\title{
Classification of Leaves Based on the Shape of Leaves Using Convolutional Neural Network Methods
}

\author{
Rizka Zulfani Syahrir ${ }^{1}$, Eri Prasetyo Wibowo ${ }^{2}$ \\ Department of Informatics ${ }^{1}$, Department of Information Technology ${ }^{2}$ \\ Universitas Gunadarma ${ }^{1,2}$
}

Komplek Mutiara Taman Palem Blok C7 No. 20, Jl. Raya Outer Ringroad, RT.7/RW.14, Cengkareng Timur, Cengkareng, RT.7/RW.14, Cengkareng Tim., Kecamatan Cengkareng, Kota Jakarta Barat, Daerah Khusus Ibukota Jakarta 11730, Indonesia ${ }^{1,2}$ e-mail: rizkazulfani@gmail.com ${ }^{1}$, eri@staff.gunadarma.ac.id ${ }^{2}$

To cite this document:

Syahrir, R. Z., \& Eri Prasetyo Wibowo. (2021). Classification of Leaves Based on the Shape of Leaves Using Convolutional Neural Network Methods. IAIC Transactions on Sustainable Digital Innovation (ITSDI), 3(1), 1-7.

DOI: https://doi.org/10.34306/itsdi.v3i1.491

\begin{abstract}
One part of the tree, namely the leaves, which grow on the branches, has several types of leaves consisting of 4 shapes, ranging from circular shapes, elongated shapes, and some even have a finger shape. Often we mistake the shapes of these leaves. This study discusses the classification of leaves based the shape of the leaf bones using the Convolutional Neural Network, which is used to classify data that has been labeled using one of the methods, namely supervised learning. The purpose of this method is to classify a variable into the variables that have been listed. The goal is to classify leaves based on leaf shape to implement a Convolutional Neural Network algorithm model for leaf classification based on bone shape, which will produce an accuracy value. Accuracy values are obtained from conducting experiments at the training and trial stages. So it can be concluded using the epochs parameter of 30 and a batch size of 128, using ReLU and Softmax activations. The results obtained for the accuracy value for training are $98.52 \%$, while the validation is $89.06 \%$. Keywords: Leaves, Supervised Learning, Convolutional Neural Network, Classification, Accuracy
\end{abstract}

\section{Introduction}

Plants play a very vital role in our life as they provide us food and oxygen. We require a good understanding and knowledge about plants and identify new and rare species to increase the agricultural productivity and also to support drug industry [1]. Plants are one of the living things, which have their parts, from the roots to the fruit. One of the charms of knowing about plants is the leaves. Leaves have many shapes, ranging from circular shapes, elongated shapes, and some even have a finger shape. Often we mistake the shapes of these leaves. To assist in identifying these leaves, an application for estimating leaves based on their bone shape was created using the Deep Learning methods.

Deep learning is a scientific field that is currently developing. Being able to study like the neural network of the human brain is an advantage of deep learning itself.. With deep learning, it can be easy to solve problems such as identifying plants based on leaf bone shape. One significant method of identifying objects is CNN (Convolutional Neural Network). Therefore, a study was conducted to classify leaves based on the shape of the leaf bones, and to 
determine the level of accuracy obtained using the CNN technique. This is what underlies the making of "Classification of Leaves Based on the Shape of Leaves Using Convolutional Neural Network Methods".

\section{Research Method}

A. Create Database

This dataset will be used during the training and test process. In preparing the dataset, there are image searches, leaf shoots and image cropping. After the dataset is created, the data preprocessing process can be carried out. After combining image search on Google Image and shooting.

Image searches to be used as train data and test data by searching for images one by one on the Google Image site. The images you are looking for are curved leaves, finger leaves, pinnate leaves, and parallel leaves. Downloaded data for the training process and test process uses the *.jpg format.

In addition to searching for images via Google Image, images are obtained by taking pictures using the Oppo A31 Smartphone. The image that is taken is done in a bright place so that the captured image produces an image that is not blurry. Pictures are taken as needed. The resulting image when shooting in *.jpg format.

The number of ready-made datasets is 2400 images. The images are divided manually for each folder, 1600 images for the training folder and 800 images for the test folder.

\section{B. Preprocessing Database}

Before carrying out the stage of building the CNN model architecture, preprocessing is carried out first, so that the required data is in accordance with the architectural requirements of the CNN model. This stage is to get better data when processed by the CNN model. Preprocessing is needed to improve image quality, preserve the edges within the image, and enhance the image [2]. What was done during the preprocessing stage was scaling and splitting the dataset. In the scaling stage, which is to align all image sizes to $128 \times 128$ pixels. To split the dataset is to solve the training dataset and validation dataset to $80 \%$ and $20 \%$ which are used to carry out the training stage.

\section{CNN Model Formation}

After the preprocessing stage is complete, then the next step is to create a Convolutional Neural Network model to classify leaves based on the shape of the leaf bones.

\section{CNN Model Training}

After the model has been created, the next step is to carry out the model training phase. This training uses a dataset that has been shared previously, namely the training dataset and the validation dataset.

\subsection{Literature Review}

A. Leaves

Leaves are one of the most important organs of plants in maintaining life, because plants are obligate autotrophic organisms that must supply their own energy needs through the conversion of sunlight into chemical energy [3]. Leaves have 4 leaf bone shapes based on bone shape, namely curved, parallel, pinnate, and fingering. [4]

\section{B. Deep Learning}

According to Yanming Guo [5] stated that deep learning is a subfield of machine learning which attempts to learn high-level abstractions in data by utilizing hierarchical architectures. It is an emerging approach and has been widely applied in traditional artificial intelligence domains, such as semantic parsing, transfer learning, natural language processing, computer vision and many more. The computer vision field has studied deep learning intensively in recent years, as a consequence, related approaches have emerged in large numbers. In general, the deep learning method consists of 4 categories according to the basic method, 
including Convolutional Neural Networks (CNNs), Restricted Boltzmann Machines (RBMs), Autoencoder and Sparse Coding. [22].

Deep Learning solves problems such as extracting high-level abstract features in representation learning by introducing representations that are expressed in other, simpler forms of representations. [6]

"Deep" in Deep Learning comes from the many layers that are built into the Deep Learning model, which is usually a Neural Network. Convolutional Neural Network (CNN) can consist of many, many layers of the model, where each layer takes input from the previous layer, processes it, and outputs it to the next layer [7].

C. Convolutional Neural Network (CNN)

CNN is a multi-layer overseeing the learning neural network. Feature based on correlation for local features, neurons in CNN only need to be extracted, then the output of all higher level neurons is combined to obtain global features, which avoids the complex process of feature extraction and data reconstruction in traditional deep learning algorithms. Better predictive results have been achieved by $\mathrm{CNN}$ in various fields for example image processing, natural language processing and medical information. For example, a level of image processing that is close to human eye recognition in image processing accuracy has been achieved by CNN based on its unique feature extraction method. [8].

Convolution layer, pooling layer, and fully connected layer are the 3 layers of CNN in general. The weight is divided by the convolution layer while the sampling function is carried out by the pooling layer for the output generated by the convolution layer and the data rate is reduced from the layer below it. Output for several fully connected layers uses the output from the pooling layer [9].

\section{Dropout Reguralization}

Dropouts, a way to avoid overfitting and also to make the learning process more agile. The dropout corresponds to eliminating neurons on the network, both hidden and visible layers. By deleting a neuron that means temporarily removing it from the network. The removed neurons will be randomly selected [10].

A dropout is defined as a neural network that is regulated by means of added interference to the hidden unit. The dropout applied to the neural network is the same as the "thin" tissue sampled. The thin network consists of all dropout survivors. [11].

E. Activation Function

The activation function is a non-linear function in Artificial Neural Networks that can convert input data into higher dimensions in order to perform simple hyperlane cuts that allow classification [12].

a. ReLU (Rectified Linear Unit)

A strong biological and mathematical basis is the activation function that ReLU introduced by. Neural training in training was enhanced by demonstrating that in 2011. The thresholding value was at 0 , namely $f(x)=\max (0, x)$ which it did. To put it simply, it issues 0 when $x<0$, and conversely, it issues a linear function when $x \geq 0$ [13].

b. Softmax

A more responsive result and better probabilistic interpretation is given by softmax, compared to other classification algorithms. Computes the probability of all labels, possible inside softmax. Softmax will take from a real vector and transform it into a vector with values between zero and one that add up to one value [14].

F. Optimizer Function

Optimizer shapes the model into the most accurate shape by adjusting the weight. Loss function is a guide to notify the optimizer when it is moving in the right or wrong direction [15]. 
G. Cross-Entropy Loss Function

In this paper, the model uses a type of loss function, namely categorical cross-entropy loss. A multi-class classification assignment using a loss function is an understanding of Categorical cross-entropy. One of the possible categories can only have an example and which model to decide which is the assignment of categorical cross-entropy [16].

H. Confusion Matrix

The confusion matrix contains information about the classification system that performs both actual and predictive classification. The system performance is usually used data in the matrix by evaluating it. For classifiers of two classes, confusion matrix will be shown in Table 1. [17].

Table 1 Confusion Matrix Overview

\begin{tabular}{|c|c|c|c|}
\hline \multicolumn{2}{|c|}{} & \multicolumn{2}{c|}{ Prediction } \\
\cline { 3 - 4 } Actual & Positive & Positive & Negative \\
\cline { 2 - 4 } & Negative & c & b \\
\hline \multirow{2}{*}{ A }
\end{tabular}

The entries in the confusion matrix have the following meaning in the context of our study:

- number of correct predictions that negative instances are a

- number of incorrect predictions that positive instances are $b$

- number of incorrect predictions that negative instances are $c$

I. Python

- number of correct predictions that examples is positive are $\mathrm{d}$

Python is an open source programming language with the intention of being a general purpose. It is optimized for software quality, developer productivity, program portability, and component integration. At least hundreds of thousands of developers around the world use python in various fields.[18].

For most compilation tasks, python is quite agile, compared to compiled low-level languages, user-friendly high-level languages are often slowed down.For the performance of critical pieces of code, combining python with specially written code, it will generate almost optimal speeds in most cases [19].

Python has a popular and excellent package manager called PIP. Using PIP you can start installing or removing Python libraries that will be or are not being used anymore [20].

\section{J. Tensorflow}

TensorFlow is used to experiment with deep learning models, train models on large datasets, and make them fit for production. In addition, TensorFlow also supports large-scale training and inference using hundreds of servers that use a Graphic Processing Unit (GPU) for efficient training [21].

\section{Findings}

A. Results of the CNN Model Training

The following are the results of training accuracy and loss from the training dataset.

Table 2 Results of Accuracy And Training Loss

\begin{tabular}{|c|c|c|}
\hline & Accuracy & Loss \\
\hline Training Dataset & 0,9852 & 0,0545 \\
\hline Validation Dataset & 0,8906 & 0,3949 \\
\hline
\end{tabular}




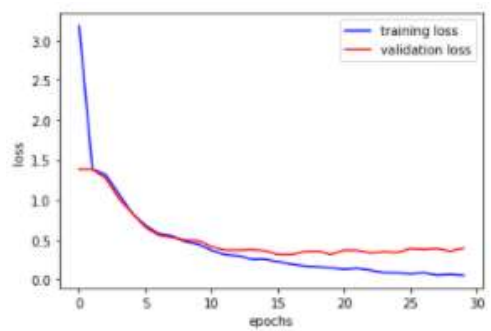

Figure 1 Graph of Training Loss Results

For the loss results, it can be seen in Figure 1 that shows a significant decrease in the training dataset and validation dataset. The training dataset has decreased significantly at epoch $0-5$ between 3.0 and 0.5 . Then the graph continues to decline, almost reaching 0.0 . The validation dataset has decreased from $0-5$ between 1.5 to 0.5 . The next validation dataset graph runs steadily until it experiences a slight increase between 20-30 epochs.

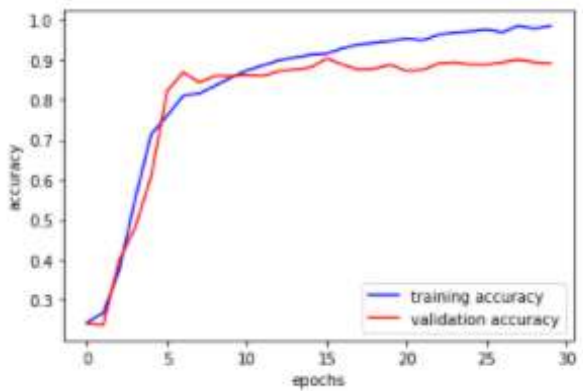

Figure 2 Graph of Accuracy Training Results

The accuracy results can be seen in Figure 2, where the training dataset and the validation dataset have increased in accuracy. The training dataset has increased in accuracy from epochs $0-5$ with an accuracy value of 0.3 to 0.8 , then the training dataset has continued to increase until it almost reaches a value of 1.0. The validation dataset has increased accuracy between epochs $0-5$ with an accuracy value of 0.3 to 0.8 .

B. Confusion Matrix Dataset Test Results

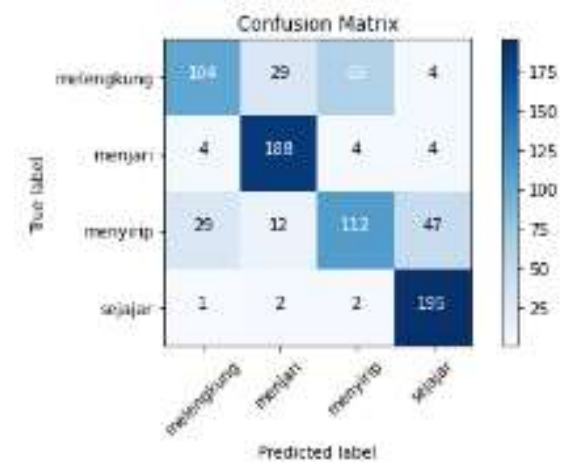

Figure 3 Results of the Confusion Matrix

Prediction visualization uses a test dataset that has been tested before. The displayed image will show a leaf image and two text above the leaf image, namely the text 'True' and the text 'Predicted'. 'True' means that the image displayed is the image in the test dataset in accordance with the shape of the leaves, while 'Predicted' means that the image displayed is an image predicted by a computer. 
Figure 4 shows the correct image and the incorrect image, which shows 4 of the 800 test datasets. Two of the four pictures predict wrong, while the other two predict right. This happens because there is ambiguity in the image. The image still has defects so it is still wrong when predicting. One example in Figure 4 is that the text 'True' contains pinnate, while 'Predicted' contains parallel, meaning that the system predicts it is wrong to define.

C. Prediction Visualization

In Figure 4 Displays the correct image and the incorrect image, which is shown 4 of the 800 test datasets. In this picture, some of them have predicted wrong, because there is ambiguity in the image. The image still has defects so it is still wrong when predicting.
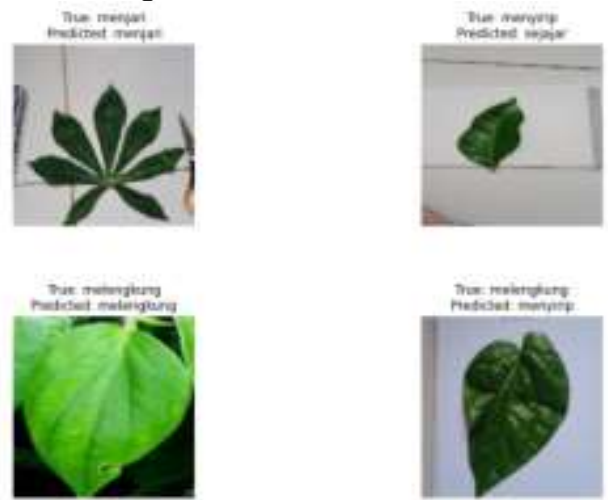

Figure 4 Prediction Visualization Results

\section{Conclusion}

In this study, he succeeded in completing the making of leaf classification based on the shape of the leaf bone using the Convolutional Neural Network (CNN) method. The CNN model has an architecture in the form of 1 input layer, 2 convolution layers, 2 pooling layers, 1 flatten layer and 1 fully connected layer. The activation functions used are ReLU and Softmax.

Implementation of the Convolutional Neural Network method with the validation dataset that has been divided by $20 \%$ from the 1600 training dataset to 1280 for the training dataset and 320 for the validation dataset. The CNN model has epochs of 20 and a batch size of 128 , which takes 7 minutes. The training dataset produced an accuracy of $98.52 \%$ and a loss of $5.54 \%$, while the validation dataset produced an accuracy of $89.06 \%$ and a loss of $39.49 \%$.

\section{Reference}

[1] Naglot, D., Kasliwal, P. S., Gaikwad, S. J., \& Agrawal, N. D. (2019). Indian Plant Recognition System Using Convolutional Neural Network. International Journal Of Computer Sciences and Engineering, 7(6), 276-280. doi:https://doi.org/10.26438/ijcse/v7i6.276280

[2] Wisudawati, L. M., Madenda, S., Wibowo, E. P., \& Abdullah, A. A. (2020). Feature Extraction Optimization with Combination 2D-Discrete Wavelet Transform and Gray Level Co-Occurrence Matrix for Classifying Normal and Abnormal Breast Tumors. Modern Applied Science, 14, 51-62

[3] Manis, S. (2019). Pengertian Daun : Fungsi, Struktur Bagian dan Jenis Daun Pada Tumbuhan. Diambil kembali dari Pelajaran https://www.pelajaran.co.id/2019/03/daun.html

[4] Aulia, K. (2017). Bentuk-bentuk Tulang Daun pada Tumbuhan Hijau. Diambil kembali dari Juragan Les: https://www.juraganles.com/2017/08/bentuk-bentuk-tulang-daun-padatumbuhan-hijau.html

[5] Guo, Y., \& al, e. (2015). Deep learning for visual understanding: A review. Neurocomputing, 1-22. doi:https://doi.org/10.1016/j.neucom.2015.09.116

[6] Goodfellow, I., Bengio, Y., \& Aaron, C. (2016). Deep Learning. MIT Press.

[7] Wehle, H.-D. (2017). Machine Learning, Deep Learning and Al: What's the Difference ? Data Scientist Innovation Day, (hal. 2-5) 
[8] Zheng, Y., Gao, Z., Wang, Y., \& Fu, Q. (2020). MOOC Dropout Prediction Using FWTSCNN Model Based on Fused Feature Weighting and Time Series. 225324-225335.

[9] Syulistyo, A. R., Purnomo, D. M., Rachmadi, M. F., \& Wibowo, A. (2016). Particle Swarm Optimization (PSO) For Training Optimization On Convolutional Neural Network (CNN). Journal of Computer Science and Information, 9(1), 52-58. doi:https://doi.org/10.21609/jiki.v9i1.366

[10] Lina, Q. (2019). Apa itu Convolutional Neural Network? Diambil kembali dari Medium: https://medium.com/@16611110/apa-itu-convolutional-neural-network-836f70b193a4

[11] Srivastava, N., Hinton, G., Krizhevsky, A., Sutskever, I., \& Salakhutdinov, R. (2014). Dropout: A Simple Way to Prevent Neural Networks from Overfitting. Journal of Machine Learning Research, 15, 1929-1958.

[12] Putra, I. W. (2016). Image Classification Using Convolution Neural Network (CNN) on Caltech 101. Undergraduate Thesis, Institut Teknologi Sepuluh Nopember, Surabaya.

[13] Agarap, A. F. (2019). Deep Learning using Rectified Linear Units (ReLU). doi:arXiv:1803.08375

[14] Ilahiyah, S., \& Nilogiri, A. (2018). Implementasi Deep Learning Pada Identifikasi Jenis Tumbuhan Berdasarkan Citra Daun Menggunakan Convolutional Neural Network. JUSTINDO(Jurnal Sistem \& Teknologi Informasi Indonesia), 3(2), 49-56. doi:https://doi.org/10.32528/justindo.v3i2.2254

[15] Team. (2020, December 26). Optimizers. Diambil kembali dari ML Glossary: https://mlcheatsheet.readthedocs.io/_downloads/en/latest/pdf/

[16] Categorical Crossentropy. (t.thn.). Diambil kembali dari Peltarion: https://peltarion.com/knowledge-center/documentation/modeling-view/build-an-aimodel/loss-functions/categorical-crossentropy

[17] Santra, A., \& Christy, C. J. (2012). Genetic Algorithm and Confusion Matrix for Document Clustering. IJCSI International Journal of Computer Science Issues, 322-328.

[18] Lutz, M. (2010). Programming Python, Fourth Edition. California: O'Reilly Media, Inc.

[19] Fanghor, H. (2015). Python for Computational Science and Engineering. Zenodo.

[20] Supardi, Y. (2017). Semua Bisa Menjadi Programmer Python Basic. Jakarta: PT Elex Media Komputindo.

[21] Pangestu, M. A., \& Bunyamin, H. (2018). Analisis Performa dan Pengembangan Sistem Deteksi Ras Anjing pada Gambar dengan Menggunakan Pre-Trained CNN Model. Jurnal Teknik Informatika dan Sistem Informasi, 4(2), 337-344.

[22] Harahap, R.A., Wibowo, E.P. and Harahap, R.K. (2020). Detection and Simulation of Vacant Parking Lot Space Using EAST Algorithm and Haar Cascade. 2020 Fifth International Conference on Informatics and Computing (ICIC), Gorontalo, 2020. 